\title{
Spinodal Decomposition of Geometrically Constrained CuNiFe Thin Films
}

\author{
J. T. McKeown,* J. D. Sugar,* V. Radmilovic,** A. M. Glaeser,* and R. Gronsky* \\ * Department of Materials Science and Engineering, University of California at Berkeley, Berkeley, CA 94720 \\ ** National Center for Electron Microscopy, Lawrence Berkeley National Laboratory, Berkeley, CA 94720
}

High-density, controlled fine-scale domain structures offer the potential to exploit materials properties that cannot be achieved in the bulk. Processing is the key to obtaining microstructural control at these finer spatial scales, and this has generally been dictated by patterning and deposition techniques. An alternative approach is to use a chemical process, such as spinodal decomposition, that leads to partitioning of an initially continuous phase to further refine the structure. By combining imposed geometric constraints with the knowledge of bulk alloy systems, spinodal decomposition can be used to obtain structures with a desired morphology, scale, and properties. The imposed geometric constraint - a free-standing thin film or a fully encapsulated thin film-can "direct" the chemical partitioning of the phases by suppression of the composition modulation in directions where the linear dimension of the confinement is smaller than the wavelength of the modulation, resulting in two-dimensional or one-dimensional fine-scale domain structures. CuNiFe alloys spinodally decompose into a ferromagnetic $\mathrm{NiFe}$-rich phase and a paramagnetic $\mathrm{Cu}$-rich phase; this bulk alloy has been characterized in prior studies [1-2]. The aim of this work was to investigate the crystallographic orientation relationship between the film and the sapphire substrate, distribution of magnetic and paramagnetic phases, and consequently define the wavelength of the composition modulation under the geometrically constrained conditions.

$\mathrm{CuNiFe}$ thin films were deposited on c-plane (0001) sapphire substrates using pulsed-laser deposition. The as-deposited films were polycrystalline with a columnar grain structure; the films had a dominant (111) texture and were heavily twinned. In order to obtain epitaxial films, the alloy was encapsulated within the sapphire by lithographic patterning, deposition, and bonding techniques. The encapsulated alloy was then melted and, upon cooling, single crystals were obtained by a nucleation-controlled liquid-phase epitaxial process with the orientation relationship: $(1 \overline{1} 1)_{\mathrm{CuNiFe}}\left\|(0001)_{\text {sapphire, }}[110]_{\mathrm{CuNiFe}}\right\|[10 \overline{1} 0]_{\text {sapphire }}$ (shown in Figure 1a). Figure $1 \mathrm{~b}$ is a phase-contrast image of the interface in the $110 \mathrm{ZA}$ of the film. The $12 \overline{1} \mathrm{ZA}$ of the film is obtained by rotating $30^{\circ}$ about the [1 $\overline{1} 1$ ] direction, clearly revealing the (1 $\left.\overline{1} 1\right)$ planes of the alloy parallel to the (0001)oriented sapphire substrate.

Figures $2 \mathrm{a}$ and $2 \mathrm{~b}$ show bright-field and weak-beam dark-field images obtained by tilting into a systematicrow orientation from the $110 \mathrm{ZA}$ of the film. The composition modulation is to some extent evident only in the weak-beam dark-field image. Energy-filtered imaging using the $\mathrm{K}$ edges of the individual elements was employed to accurately measure the wavelength of this modulation. The jump-ratio images of the $\mathrm{Cu}, \mathrm{Ni}$, and Fe given in Figures 3a, 3b, and 3c respectively show that the encapsulated alloy decomposed with an average wavelength of approximately $160 \mathrm{~nm}$ after annealing $20 \mathrm{~h}$ at $700^{\circ} \mathrm{C}$. This is roughly 2.5 times larger than the wavelength observed in a bulk CuNiFe alloy of the same composition after the same heat treatment [1]. As in bulk $\mathrm{CuNiFe}$ alloys, the composition modulations are in the $<100>$ directions.

Future investigations include the effects of strain and interfacial energetics on early-stage atomic redistribution in constrained volumes, followed by the complete evolution of the composition modulation with time at temperature. This process holds promise and potential to fabricate controlled two-dimensional nanoscale domain structures [4].

\section{References}

[1] E. P. Butler and G. Thomas, Acta Metall. 18, 347 (1970).

[2] R. Gronsky and G. Thomas, Acta Metall. 23, 1163 (1975). 
[3] This research was supported by the Director, Office of Science, Office of Basic Energy Sciences, Materials Science and Engineering Division of the U.S. Department of Energy under Contract No. DE-FG02-02ER46008 and Contract No. DE-AC02-05CH11231.
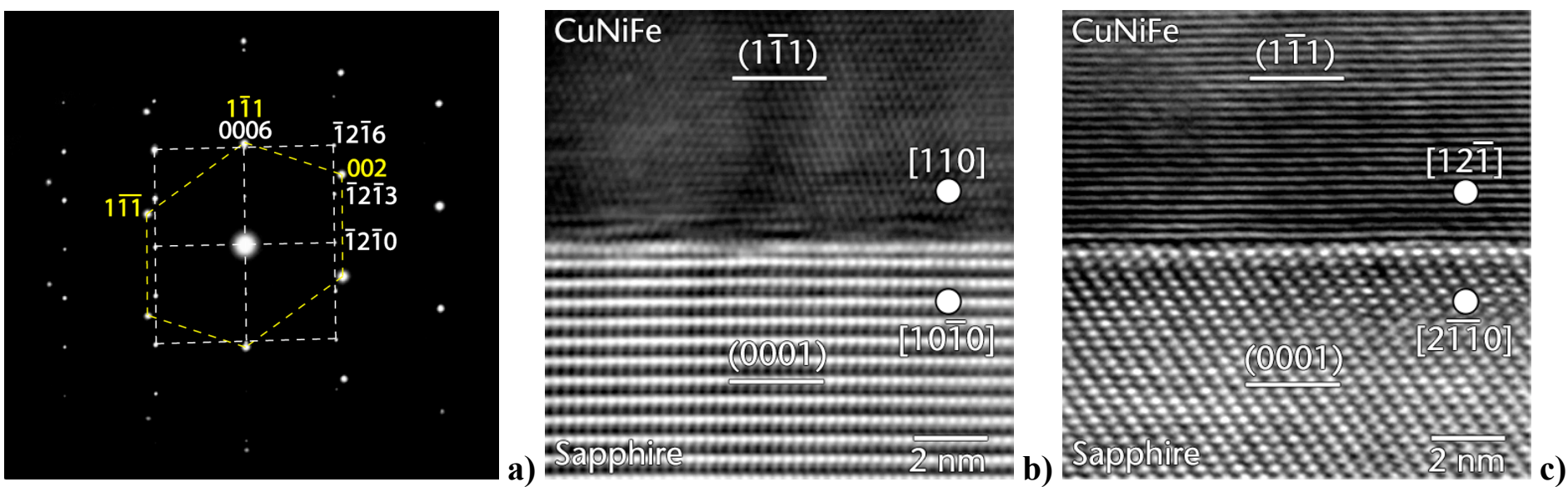

Figure 1: a) Diffraction pattern of alloy-sapphire interface in $110 \mathrm{ZA}$ of the film. Phase-contrast images of interface in b) $110 \mathrm{ZA}$ and c) $12 \overline{1} \mathrm{ZA}$ of the film, showing the (1 $\overline{1} 1)$ planes parallel to the (0001)-oriented surface of the sapphire substrate.

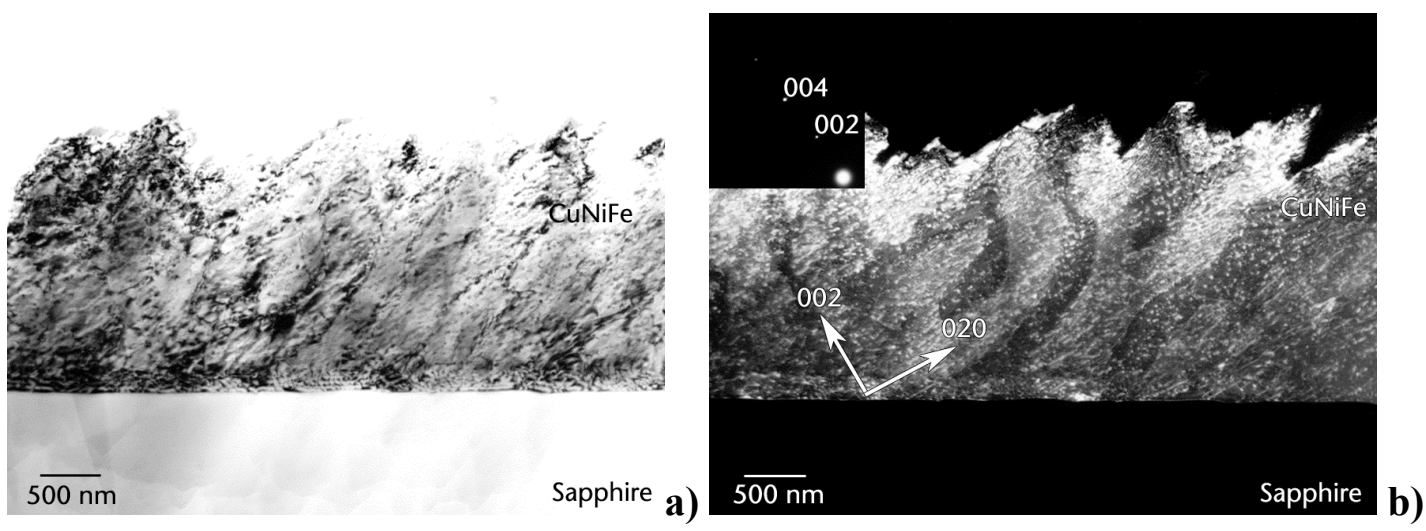

Figure 2: a) Bright-field and b) corresponding weak-beam dark-field TEM images showing composition modulation in $<002>$ directions.
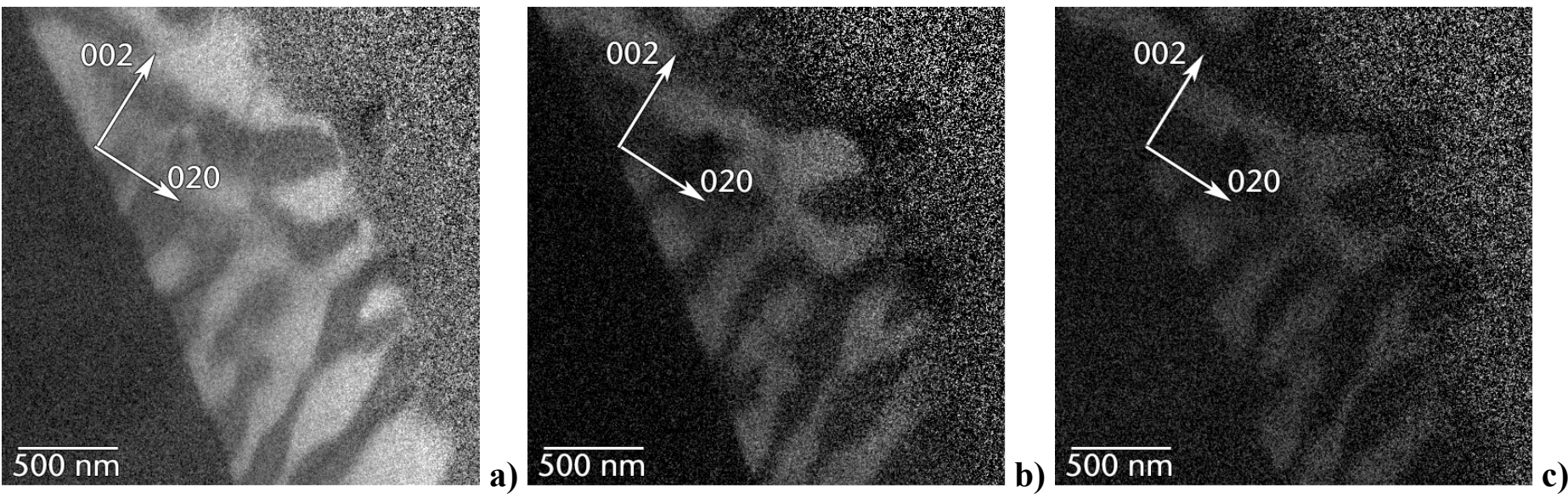

Figure 3: Jump-ratio images showing composition modulation of a) $\mathrm{Cu}, \mathrm{b}$ ) $\mathrm{Ni}$, and c) $\mathrm{Fe}$. 